\title{
Cosmic ray acceleration and nonthermal emission from ultra-fast outflows in active galactic nuclei
}

\author{
Susumu Inoue ${ }^{* \dagger}$ \\ RIKEN, Wako, Japan \\ E-mail: susumu.inoue@riken.jp \\ Ruo-Yu Liu \\ Max-Planck-Institut für Kernphysik, Heidelberg, Germany \\ E-mail: Ruoyu Liu <liuruoyu1986@gmail.com>
}

\section{Kohta Murase}

Penn State University, USA

E-mail: muraseepsu.edu

\begin{abstract}
X-ray observations of active galactic nuclei (AGN) are revealing the widespread existence of ultra-fast outflows, which are powerful outflows of baryonic material approaching mildly relativistic velocities, seen as variable, blue-shifted absorption lines of ionized heavy elements [1]. Being observed in both radio-quiet [2] and radio-loud [3] AGN, they are distinct from the better studies radio jets. Plausibly interpreted as winds driven the accretion disk, their interaction with their environment may be the key cause of known correlations between the properties of supermassive black holes and their host galaxies [4].

We discuss the possibility of acceleration of electrons and hadrons in collisionless shocks that are likely to form at different locations in such outflows, including that of ultra-high-energy cosmic rays. We also present expectations for the consequent nonthermal emission from the radio band up to high-energy gamma-rays, in particular synchrotron and inverse Compton emission from accelerated electrons. These are compared with existing data on selected objects of interest such as the radio-quiet object ESO 323-G77 [5] (Fig 11) and the radio-loud object 3C 120 [6] (Fig] More details on the modeling, together with prospects for further observations with current and future instruments will be addressed at the conference and in a updated version of this proceedings paper.
\end{abstract}

The 34th International Cosmic Ray Conference

30 July- 6 August, 2015

The Hague, The Netherlands

\footnotetext{
${ }^{*}$ Speaker.
} 


\section{Figures}

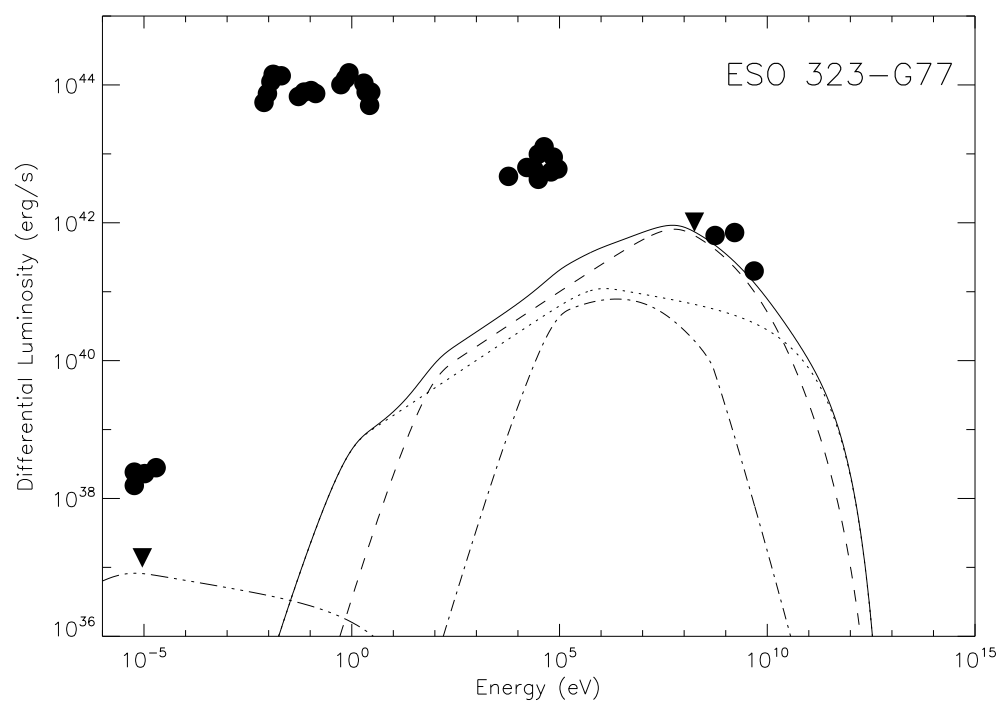

Figure 1: Broadband spectra for the radio-quiet AGN ESO 323-G77, compared with a model of emission from electrons accelerated in UFO shocks with total power $L_{e}=10^{43} \mathrm{erg} / \mathrm{s}$. The dotted, dashed and dashdotted curves correspond to inverse Compton upscattering of external infrared, UV and X-ray photons from the nucleus, while the long dash-dotted curve is synchrotron emission.

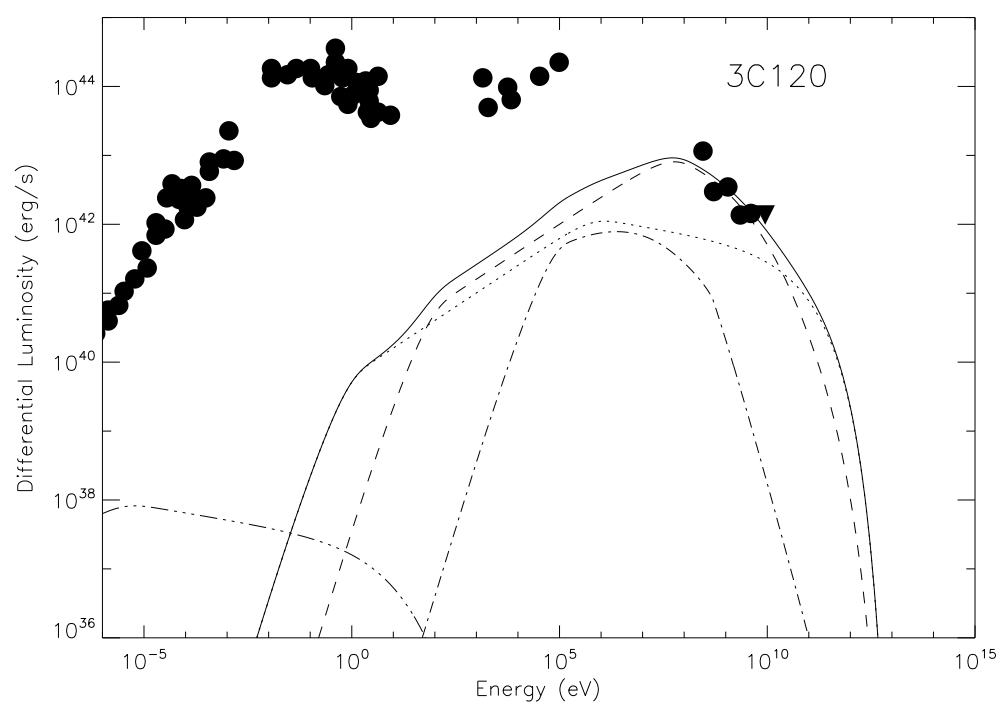

Figure 2: Broadband spectra for the radio-loud AGN 3C 120, compared with a model of emission from electrons accelerated in UFO shocks with total power $L_{e}=10^{44} \mathrm{erg} / \mathrm{s}$. The dotted, dashed and dash-dotted curves correspond to inverse Compton upscattering of external infrared, UV and X-ray photons from the nucleus, while the long dash-dotted curve is synchrotron emission. 


\section{References}

[1] K. Pounds, Space Sci. Rev. 183, 339, 2014

[2] F. Tombesi, M. Cappi, J. N. Reeves, V. Braito, MNRAS, 422, L1, 2012

[3] F. Tombesi et al., MNRAS, 443, 2154, 2014

[4] C. Faucher-Giguère, E. Quataert, MNRAS, 425, 605, 2012

[5] M. Ackermann et al., ApJ, 747, 104, 2012

[6] J. Kataoka et al., ApJ, 740, 29, 2011 